\title{
Nuclear Fusion during Early Stage of Microspore Embryogenesis Indicates Chromosome Doubling in Wheat (Triticum aestivum)
}

\author{
Roland Griggs, Ming Y. Zheng* \\ Department of Biology, Gordon College, Wenham, MA, USA \\ Email: "ming.zheng@gordon.edu
}

Received 21 January 2016; accepted 13 March 2016; published 17 March 2016

Copyright (C) 2016 by authors and Scientific Research Publishing Inc.

This work is licensed under the Creative Commons Attribution International License (CC BY). http://creativecommons.org/licenses/by/4.0/

\section{Abstract}

Studies of barley and maize indicate that chromosome doubling occurs via nuclear fusion during an early stage of microspore embryogenesis, but the time and mechanism by which chromosome doubling occurs in bread wheat (Triticum aestivum) remains undetermined. The purpose of this study was to determine the relative time during induction culture when chromosome doubling may occur in wheat, and to identify early indicators for doubled haploid microspores. Microspore nuclei were stained with 4',6-diamidino-2-phenylindole (DAPI) and observed under a fluorescent microscope on the day of isolation, three days after isolation, and six days after isolation. The change in the percentage of microspores containing a single small nucleus, two small nuclei, a single enlarged nucleus, and three or more nuclei was then tracked throughout the six-day period. Ploidy levels were estimated by determining the cross-sectional area and number of nucleoli in microspores containing small and large nuclei then comparing the results of each respective celltype. The percentage of microspores containing enlarged nuclei increased throughout the six-day test period, and the percentage of binucleated microspores containing small nuclei decreased. Comparison of the changes in average percentage of microspores containing a single small nucleus, binucleated microspores, microspores containing a single large nucleus, and multinucleate microspores on days 0,3 , and 6 indicates that nuclei classified as "small" are likely haploids and nuclei classified as "large" are doubled haploids. The percentage of microspores with enlarged nucleus (nuclei) during the first six days of induction culture could be used as an early indicator for the frequency of chromosome doubling in wheat microspore culture.

\footnotetext{
${ }^{*}$ Corresponding author.
} 


\section{Keywords}

\section{Cell Culture, Chromosome Doubling, Crop Breeding, Doubled Haploid (DH), Embryoids, Microspore Embryogenesis (ME), Wheat}

\section{Introduction}

After microspores are induced for embryogenesis, they undergo various morphological changes that distinguish them from non-embryogenic microspores. Some of the first indicators of embryogenic microspores include enlargement of microspores [1] and nuclear migration towards the center of the cell caused by rearrangement of the cytoskeleton [2]. The large central vacuole breaks into smaller vacuoles located around the periphery of the cell and intersected by strands of cytoplasm that stretch from the center to the perimeter of the cell thus forming a star-like structure [3]-[5]. Researchers have observed this star-like morphology in numerous plant species including wheat [3] [5] [6], barley [7], tobacco [8], and rapeseed [9]. Because of these findings, the star-like structure serves as one of the most recognizable features of early-stage embryogenic microspores. As embryogenic microspores continue to develop, they undergo cellular divisions within the exine wall thus forming multicellular structures [4]. The exine wall ruptures as pre-embryoids emerge, and regenerable embryoids form at the end of development. Shortly after the embryoids become visible (approximately 28 days in culture), they can be transferred onto the regeneration medium where they will "germinate" into plants, either haploids or doubled haploids (DH) [10].

Studies in wheat and barley have shown high percentages of DH production in response to ME induction [3] [11]-[13]. If plants are completely DH, then chromosome doubling must occur early during development; otherwise, many plants would generate chimeric tissues (i.e. parts of the plant would be haploid and other parts DH). According to Kasha et al. [14], chromosome doubling most likely occurs as a result of endoreduplication or nuclear fusion. Endoreduplication is a process in which DNA replication occurs in the absence of mitosis thereby increasing the chromosome number within cells [15]. Nuclear fusion, on the other hand, occurs when separate nuclei within the same microspore fuse to form a new nucleus or new nuclei. Several mechanisms for nuclear fusion have been proposed. Sunderland et al. [16] note increases in chromosome number in Daturaoccur through both endoreduplication and nuclear fusion. Moreover, a combination of nuclear fusion and endoreduplication may lead to plantlets with ploidy levels greater than $2 \mathrm{n}$.

Sunderland [17] reports nuclear fusion occurring when two separate nuclei, a generative nucleus and a vegetative nucleus, simultaneously begin to divide and form a shared metaphase plate. Since both dividing nuclei share the same metaphase plate, they fuse, thus creating two new nuclei with a doubled chromosome number. Chen et al. [18] report a different mechanism in which separate nuclei in barley fuse during interphase thus creating a single nucleus. Other studies also show nuclear fusion serves as the mechanism by which chromosome doubling takes place in maize [19] and barley [14] [20] [21].

Researchers have conducted numerous studies of bread wheat (Triticum aestivum L.) attempting to optimize culture conditions [22] [23] and pretreatment protocols [3] [5] [6] [11] to increase the DH yields, but they must wait until embryogenic microspores develop into plantlets before determining the percentage of DHs produced. This process can take 9 - 11 months before completion; therefore, the discovery of an early indicator of chromosome doubling would reduce the time needed to determine the effectiveness of new treatment protocols.

Using fluorescent microscopy and cells stained with 4',6-diamidino-2-phenylindole (DAPI), Kasha et al. [14] studied chromosome doubling in barley and found fused DH nuclei appear larger than haploid nuclei. They found that uninucleate cells with enlarged nuclei contained substantially more DNA than uninucleate cells with small nuclei. Another study offers further evidence supporting the correlation between enlarged nuclei and increased ploidy level. González-Melendi et al. [20] stained cells with DAPI and used confocal microscopy to measure the fluorescent intensity, sizes of nuclei, and number of nucleoli in each cell. They then used these criteria to determine the ploidy levels of cells with different nuclear sizes. They found cells with enlarged nuclei had a ploidy level twice than that of cells with small nuclei.

As of yet, no studies have been conducted to explore the mechanism of chromosome doubling in wheat. If wheat follows a chromosome doubling mechanism similar to that of barley, then enlarged nuclei may also serve 
as an early indicator of chromosome doubling in wheat. The primary objective of this study, therefore, is to track the changes in percentages of microspores with various nuclear sizes in an attempt to establish a possible correlation between these changes and chromosome doubling. The nuclear volume and the number of nucleoli from different types of microspores were also measured and compared to establish the correlation between these parameters and chromosome doubling.

\section{Materials and Methodology}

\subsection{Basic Protocol for Microspore Cultures}

Methods for growing wheat plants, collecting tillers, pretreatment, isolation, and plant regeneration were adopted from Zheng et al. [10] [23].

\subsection{Viability Assay}

Immediately following isolations, $20 \mu \mathrm{L}$ of fluoresce in diacetate (FDA) working solution was mixed with 20 $\mu \mathrm{L}$ of isolated microspore culture. The solution containing microspores and FDA was then fixed to a slide, and a $4 \times 4$ grid comprised of approximately equal sized squares was drawn on a coverslip using a permanent marker. The coverslip was then placed on the slide and sealed with clear nail polish. Microspores were observed using an Olympus IX81 inverted fluorescent microscope fixed with a Retiga-SRV Fast 1394 camera (Q Imaging, Canada). Live microspores fluoresced green, and the percentage of live microspores was calculated by counting then dividing by the total number of microspores per slide. If cell density was low, all microspores within at least eight of the grid squares were counted. If cell density was high, a small area within eight to twelve of the sixteen squares was selected and microspores within that section were counted.

A minimum of 300 microspores were counted per slide whenever possible, because this number generally gave a good representation of the ratio of live to dead cells on the entire slide. A second viability assay was then conducted six days after isolation to ensure viability remained high enough to collect meaningful data. Only data from samples that produced embryoids and displayed a final viability greater than $4 \%$ were analyzed in this study, because samples with a final viability below $4 \%$ did not produce embryoids.

\subsection{Assessment of Number and Size of Nuclei via Fluorescent Microscopy}

Nuclear division and fusion were tracked over a six-day period starting the day of isolation, day 0 . On day 0,3 , and $6,20 \mu \mathrm{L}$ of cell solution was mixed with $200 \mu \mathrm{L}$ of Carnoy fixative and stored at $4^{\circ} \mathrm{C}$ for $1-3$ days [14]. After cells had been stored for at least $24 \mathrm{~h}$, a separate solution containing $2.5 \mu \mathrm{L}$ of DAPI stock solution and $97.5 \mu \mathrm{L}$ of citrate phosphate buffer was prepared then mixed with the solution containing the microspores. Immediately after mixing, the solution was centrifuged at approximately $1400 \mathrm{rpm}$ for $15 \mathrm{~s}$, and the pellet was removed and fixed to a slide using a coverslip with a $4 \times 4$ grid drawn on using a permanent marker.

When viewing the cells under the microscope, the total number of uninucleate microspores with small nuclei, uninucleate microspores with enlarged nuclei, bi-nucleate microspores, and multinucleate (3+ nuclei) microspores were counted (Figure 1), and the percentage of microspores in each respective stage was determined. Nuclei less than 1/6 the size of their respective host cells were considered small, and nuclei greater than 1/6 the size of their respective host cells were considered large. Nuclear size was determined via visual estimation, and no distinction between small and large nuclei was made in binucleate and multinucleate cells for the first three samples. These sizes were used to differentiate between large and small nuclei, because the majority of the nuclei considered small appeared approximately $1 / 4$ to $1 / 2$ the size of nuclei designated as large.

All sections and figures referring to the percentage of microspores within a given category reflect a portion of nucleated microspores unless otherwise specified. Cells devoid of nuclei were not included in most analyses because they were presumed to be dead.

After the third isolation, some binucleated and multinucleated cells containing one or more enlarged nuclei were observed. Consequently, binucleated and multinucleated cells with small nuclei were distinguished from binucleated and multinucleated cells with one or more large nuclei when recording each cell type. Figure 2 compares binucleated and multinucleated cells containing small and large nuclei.

Minitab version 14.12 (2004) was used to run statistical analyses of the percentage of cells in each category during each successive day. Statistical tests were only used to analyze the percentage of cells containing a single 

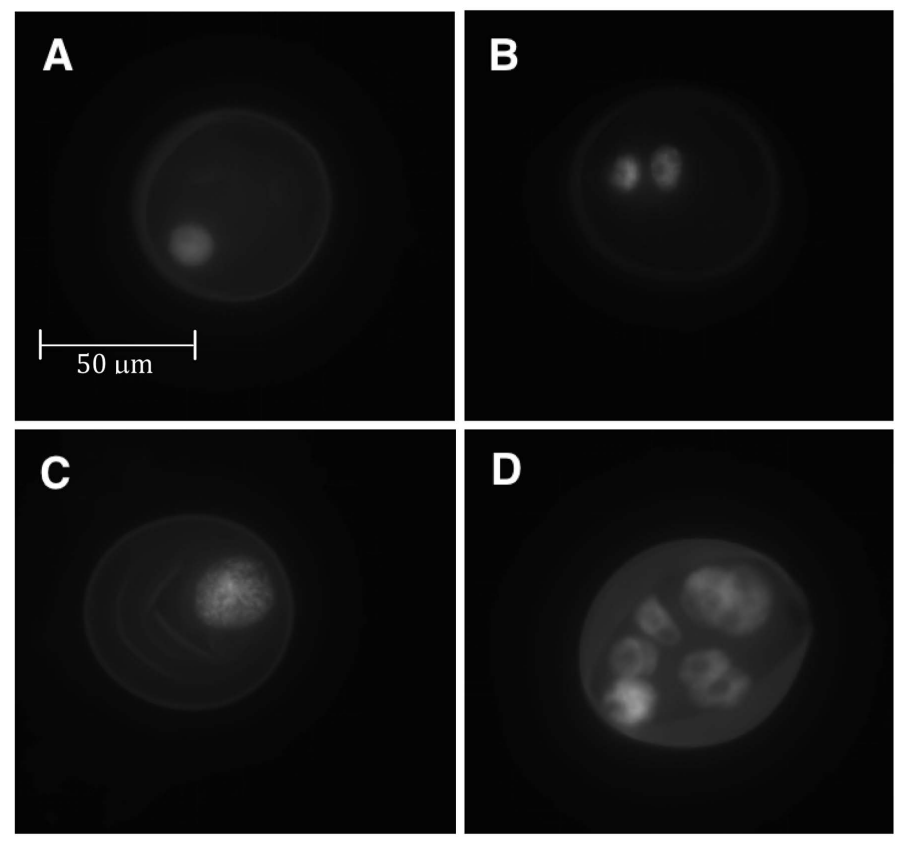

Figure 1. Comparison of cell containing single small nucleus (A), binucleate cell (B), cell containing single enlarged nucleus (C), and multinucleate cell. Nuclei stained with DAPI.
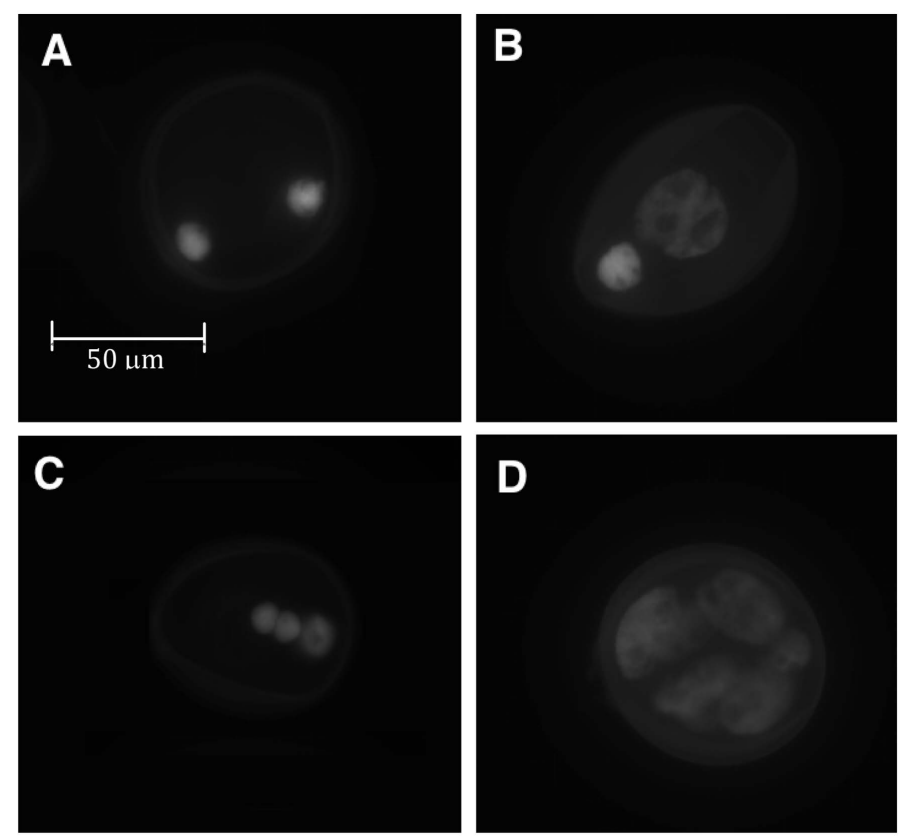

Figure 2. Comparison of binucleated microspore with small nuclei (A), binucleated microspore with one enlarged nucleus (B), multinucleated microspore with small nuclei (C), and multinucleated microspore with several enlarged nuclei. Nuclei stained with DAPI.

small nucleus, binucleated cells, multinucleated cells, and cells containing a single enlarged nucleus. Due to the small number of samples in which we differentiated between binucleated cells containing small and enlarged nuclei, all binucleated cells were placed in the same category when conducting statistical analyses. Multinucleated cells containing small nuclei and multinucleated cells containing enlarged nuclei were also grouped to- 
gether in the same way for the same reason.

In order to test the hypothesis that number of cells classified in each nucleus category does not change over time, we use the Wilks' MANOVA test [24] to compare all cell types during each day. We then used an analysis of variance test to determine whether or not each of the four nucleus categories differed individually over the course of the three time periods. For all analyses, we used an alpha level of 0.05 . If the $P$-value was lower than the alpha, we rejected our null hypothesis that days did not differ.

\subsection{Determining Area of Nuclei and Number of Nucleoli Using Confocal Microscopy}

Prior to determining the area and number of nucleoli in microspore nuclei, microspores were stained with DAPI and fixed to a slide using the same protocol described earlier. The total area of large and small nuclei was determined and the number of nucleoli for each respective nucleus type was counted using an IX81 confocal microscope (Olympus Co., Tokyo) equipped with Fluoview 3.1 software and a $405 \mathrm{nM}$ laser. To determine the area of nuclei, Z-series optical sections spaced $0.37 \mu \mathrm{m}$ apart were obtained at $60 \mathrm{X}$ objective from 35 uninucleate microspores each containing small nuclei. The microspores were collected from two separate samples, 20 microspores from one sample and 15 from another, on day 0 . The perimeters of the selected nuclei were then outlined as regions of interest using the Fluoview software, and the area within the regions of interest were automatically calculated. This same process was repeated on day 6 to determine the area of 20 uninucleate microspores containing large nuclei from the first sample (viability for the second sample was too low for analysis). Finally, the average area of the small nuclei from day 0 were calculated and compared to the average area of the large nuclei from day 6 .

After determining the area of the nuclei, nucleoli were counted within each of the 55 selected nuclei. Nucleoli appear as dark holes against the stained background of the nucleus as shown in Figure 3 [25]. Nucleoli were often difficult to identify, because many nuclei contained dark regions that looked similar to nucleoli and some nucleoli appeared faded on digital images. Consequently, we assigned nucleoli to one of three categories to indicate the level of confidence that a dark region actually depicts a nucleolus: unclear (U), somewhat clear (SC), and clear (C). Clear nucleoli generally appeared on images depicting DAPI fluorescence and on images in which DAPI fluorescence and bright field pictures were superimposed. Conversely, SC nucleoli typically only appeared on images depicting DAPI fluorescence but not on DAPI fluorescence/bright field superimposed images. Clear images also displayed little to no clouding in at least one optical section of the Z-series, whereas SC nucleoli often appeared cloudy on all optical sections. Figure 4 shows the difference between a typical SC nucleolus and three $\mathrm{C}$ nucleoli.

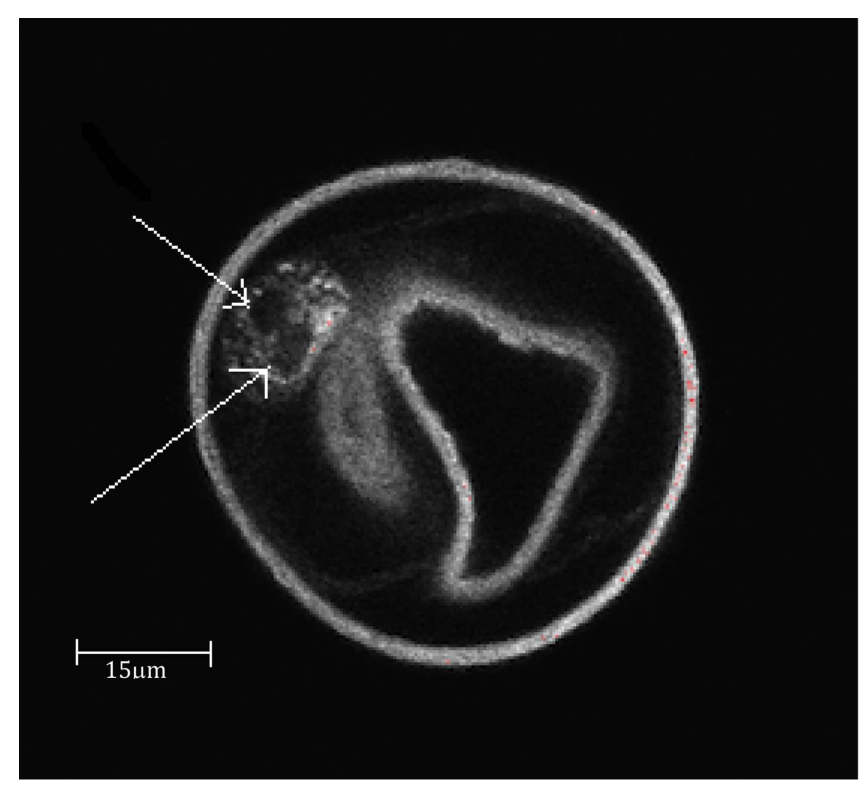

Figure 3. Microspore with small nucleus stained with DAPI. Arrows indicate two nucleoli within the nucleus of this microspore. 


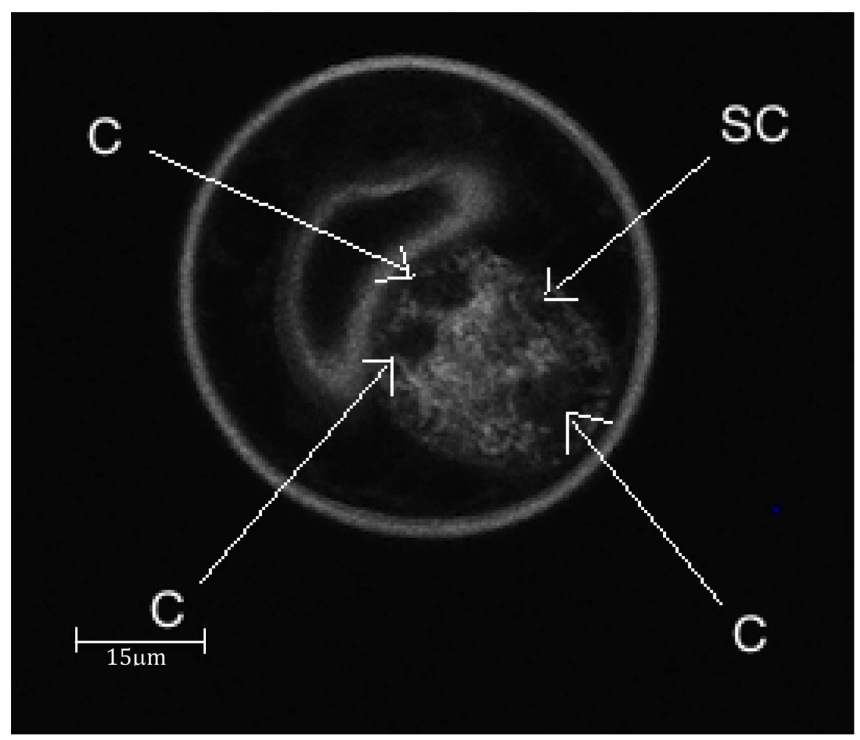

Figure 4. Image depicting three clear (C) nucleoli and one somewhat clear (SC) nucleoli. Nuclei stained with DAPI.

Nucleoli categorized as U displayed significant clouding and were difficult to distinguish from non-nucleolar dark regions. Also, because some nuclei contained dark non-nucleolar regions, we considered a dark region a nucleolus only if it had diameter of approximately one half the radius of its host nucleus or larger. Darkened regions that appeared clear but had a diameter smaller than one half the size of their host nucleus' radius were either categorized as unclear or they were not counted at all. After counting the number of nucleoli in each nucleus, we calculated the average number of $\mathrm{C}$ and SC nucleoli in small nuclei and the average number of $\mathrm{C}$ and SC nucleoli in enlarged nuclei and compared the two values. Nucleoli classified as U were not included in the averages.

\section{Results}

\subsection{Viability Assays}

A total of 25 isolations were performed over a seven-month period, but only seven of the 25 displayed high enough cell density and initial and final viability levels to provide meaningful data. Table 1 shows the percentage of live cells and the total number of cells counted to obtain these percentages on days 0 and 6 for samples 7 , $8,13,15,16,18$, and 22. Sample 18 displayed the lowest initial viability of the seven samples (16.6\%), and sample 15 displayed the highest initial viability (38.0\%). Sample 15 displayed the lowest final viability (4.3\%), while sample 13 had the highest final viability (18.7\%). The number of cells counted per sub-sample to obtain these values for initial or final viability ranged from 379 to 1333 . The number counted varied depending on the number of cells on a slide.

Non-nucleated cells were included in the counts of two of the samples that produced embryoids, samples 7 and 8, which allowed us to compare the percent viability in these samples to the number of non-nucleated cells. The percentage of non-nucleated cells, percentage of nucleated cells, total number of cells, and percent viability on day 0 and 6 for samples 7 and 8 are shown in Table 2. This data shows that the number of non-nucleated cells increases between days 0 and 6 for both samples, and the percent viability is lower than both the percentage of nucleated cells and the percentage of non-nucleated cells in both samples.

\subsection{Assessment of Number of Nuclei of Various Sizes per Cell}

Figure 5 compares the change in average percent of all four categories of cells throughout the six-day observation period. The percentage of SSN microspores (Figure 5) remained relatively steady throughout the six-day observation period with a slight decrease between days 3 and 6 . This decrease was not statistically significant. The percentage of BN microspores steadily decreased throughout the six-day observation period (Figure 5) and 
Table 1. Percentage of live cells in usable samples on day 0 (initial viability) and day 6 (final viability). The total number of cells counted for each sample is given in parentheses.

\begin{tabular}{ccc}
\hline Sample \# & Initial Viability & Final Viability \\
$\mathbf{7}$ & $30.2 \%(582)$ & $16.6 \%(416)$ \\
$\mathbf{8}$ & $17.1 \%(531)$ & $11.0 \%(1073)$ \\
$\mathbf{1 3}$ & $34.9 \%(807)$ & $18.7 \%(1333)$ \\
$\mathbf{1 5}$ & $38.0 \%(379)$ & $4.3 \%(775)$ \\
$\mathbf{1 6}$ & $28.6 \%(507)$ & $9.0 \%(502)$ \\
$\mathbf{1 8}$ & $16.6 \%(574)$ & $11.0 \%(518)$ \\
$\mathbf{2 2}$ & $26.1 \%(440)$ & $16.1 \%(529)$ \\
\hline
\end{tabular}

Table 2. Percent non-nucleated cells, percent nucleated cells, total number of cells, and percent viability on day 0 and 6 for samples 7 and 8.

\begin{tabular}{cccccc}
\hline Sample & Day & Non-nucleated & Nucleated & Total Number of Cells & \% Viability \\
\hline 7 & 0 & $7.5 \%$ & $92.5 \%$ & 267 & $30 \%$ \\
& 6 & $59.7 \%$ & $40.3 \%$ & 357 & $16.6 \%$ \\
8 & 0 & $39.6 \%$ & $60.4 \%$ & 333 & $17 \%$ \\
& 6 & $61.5 \%$ & $38.5 \%$ & 585 & $11 \%$ \\
\hline
\end{tabular}

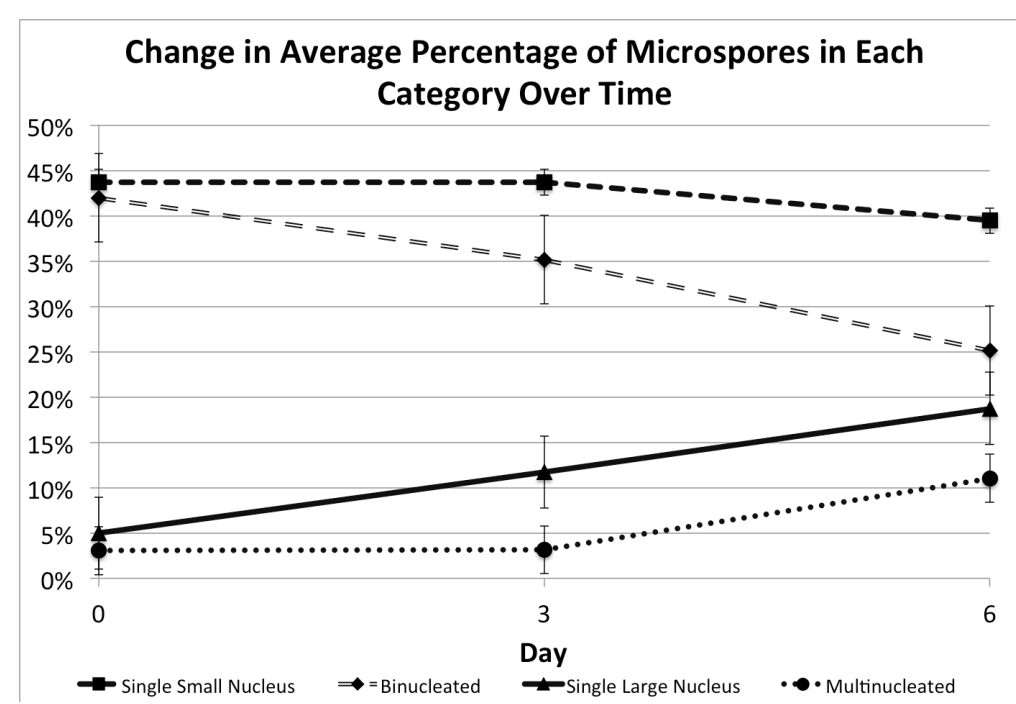

Figure 5. Comparison of the change in average percentage of microspores containing a single small nucleus, binucleated microspores, microspores containing a single large nucleus, and multinucleate microspores on days 0,3 , and 6 . Bars indicate standard error.

showed a statistically significant decrease in average percentage between day 0 and day 6 . The percentage of SLN microspores steadily increased throughout the six-day observation period (Figure 5) and also showed a statistically significant increase in average percentage between days 0 and 6. The MN microspores did not show a statistically significant change in percentage between any two specific days (Figure 5), but the difference between days 0 and 6 and between days 3 and 6 fell just outside the threshold of statistical significance. Consequently, the difference between days 0 and 6 , when multiplied by the difference between days 3 and 6 , was significant.

Results of the Wilks' MANOVA indicate that each category differs over time. The results of the analysis of variance test showed cells containing a single small nucleus (SSN) did not differ significantly over time; how- 
ever, the binucleated cells (BN), cells containing a single large nucleus (SLN), and multinucleated cells (MN) did differ significantly over the time tested. The F- and P-values for each sample are shown in Table 3.

\subsection{Nuclear Area and Number of Nucleoli in Large and Small Nuclei}

Table 4 shows the average cross-sectional area and average number of nucleoli for small and large nuclei. The cross-sectional area of an average large nucleus is approximately twice the cross-sectional area of an average small nucleus. Similarly, the average large nucleus contains 2.2 times more nucleoli than the average small nucleus. Small nuclei ranged in area from $75.2 \mu \mathrm{m}^{2}$ to $162.2 \mu \mathrm{m}^{2}$ with a standard deviation of 32.7. Large nuclei ranged in area from $131.0 \mu \mathrm{m}^{2}$ to $364.9 \mu \mathrm{m}^{2}$ with a standard deviation of 69.14 . Some small nuclei contained no visible nucleoli, and no small nuclei observed contained more than two C or SC nucleoli. Large nuclei contained between one and four C or SC nucleoli.

\section{Discussion and Conclusion}

\subsection{Viability Assay}

Because the percent viability was lower than the percentage of nucleated microspores in samples 7 and 8 (Table 2), many nucleated microspores must have been dead. Since there were relatively few SLN and MN microspores on day 0 , and since the percent of SLN and MN microspores increased over time while the percentage of SSN and BN microspores decreased, most of the dead but nucleated microspores were likely SSN and BN microspores. Consequently, most of the SLN and MN microspores were likely alive.

\subsection{Nuclear Fusion Likely Serves as the Primary Mechanism for Chromosome Doubling}

Throughout this study, the average percentage of SSN microspores remained relatively steady, and the average percentage of BN microspores decreased (Figure 5). These data suggest most BN microspores likely arise as nuclei in uninucleate microspores divide following patterns associated with normal pollen development. If BN microspores arise as a result of nuclear divisions associated with normal pollen development, then the two daughter nuclei are likely generative and vegetative nuclei produced sometimes during pretreatment. If BN microspores formed after pretreatment, then we would expect to see the percentage of SSN microspores decrease and the percentage of BN microspores increase simultaneously. Because the average percentage of SSN microspores remained stable over time, except for a slight decrease on day 6 likely caused by degeneration of dead SSN microspores, and because the average percentage of BN microspores decreased, most BN microspores likely formed before the end of pretreatment. This data indicates no significant association between the percentage of SSN and BN microspores, but we do see a significant association between the decrease in BN and the increase in SLN microspores.

Table 3. Results of analysis of variance test showing F- and P-values for cells containing a single small nucleus (SSN), a single large nucleus (SLN), binucleated cells (BN), and multinucleated cells (MN).

\begin{tabular}{ccc}
\hline Cell Type & F-Value & P-Value \\
\hline SSN & 0.32 & 0.728 \\
BN & 7.95 & 0.003 \\
SLN & 5.52 & 0.014 \\
MN & 4.09 & 0.034 \\
\hline
\end{tabular}

Table 4. Average cross-sectional area and number of nucleoli in small and large nuclei.

\begin{tabular}{ccc}
\hline & Large & Small Nuclei \\
Nuclei & $221.7 \mu \mathrm{m}^{2}$ & $107.4 \mu \mathrm{m}^{2}$ \\
Average Cross-sectional Area & 1.29 \\
Average Number of Nucleoli & 2.85 \\
\hline
\end{tabular}


Figure 5 shows the average percentage of SLN microspores increases as the average percentage of BN microspores decreases. If we compare the average percent decrease in BN microspores to the average percent increase in SLN microspores, we find BN microspores decrease at a rate similar to the rate of increase in SLN microspores. Between days 0 and 3, BN microspores decreased by an average of $6.82 \%$, while SLN microspores increased by an average of 6.73\%. Between days 3 and 6, BN microspores decreased by an average of 10.05\%, and SLN microspores increased by an average of $7.02 \%$.

This data suggests that the nuclei within BN microspores likely fuse thus producing the enlarged nuclei found in SLN microspores. If BN microspore nuclei had not fused, then we would expect the change in percentage of BN microspores to mimic that of SSN microspores, although some may also develop into MN microspores. Since the percentage of SSN microspores remained relatively constant while the percentage of BN microspores decreased and SLN microspores increased, we can attribute the increase in SLN microspores to nuclear fusion. This data is in line with previous observations made in barley [18] and wheat [11]. The data shown in Table 3 further support this conclusion and also indicate the enlarged nuclei of SLN microspores are doubled haploid.

Table 3 shows SLN nuclei contain more than twice as many nucleoli on average as SSN microspores, and the average cross-sectional area of SLN cells is twice of that of SSN microspores. This data indicates that the ploidy level of SLN microspores is likely twice of that of SSN microspores, and these results resemble those obtained in González-Melendi et al.’s [26] study of barley microspore nuclei, in which they used nuclear size and number of nucleoli as indicators of ploidy level. Nuclear size has also been described as an indicator of ploidy level in endoreduplicating root xylem vessels in wheat [25]; thus providing further evidence for the reliability of this method. Flow cytometry was not considered for ploidy analysis, because the autofluorescent exine of early-stage microspores reduced the effectiveness of this method [26].

In short, we have determined that BN microspores in early stage are likely haploid and the nuclei from BN microspores likely fuse to produce SLN microspores. We have also determined that nuclei from SLN microspores are likely doubled haploid, because they contain twice as many nucleoli, and they are substantially larger than nuclei from BN and SSN microspores. If BN microspore nuclei are haploid, if BN microspores fuse to form SLN microspores, and if SLN microspores are doubled haploid, then nuclear fusion must produce doubled haploid microspores. This study therefore shows that chromosome doubling in wheat likely occurs via nuclear fusion, but can we designate nuclear fusion as the main mechanism for producing doubled haploids? Chromosome doubling via other mechanisms such as endoreduplication may account for the large cross-sectional area of SLN microspores, but these mechanisms do not account for the decrease in BN microspores over time or the presence of additional nucleoli in SLN microspores. Consequently, nuclear fusion likely serves as the main mechanism for chromosome doubling.

If we revisit the subject of the rate of change percentage in $\mathrm{BN}$ and SLN microspores, and we compare the respective percentage of decrease and increase in BN and SLN microspores between days 3 and 6, we find a $3.03 \%$ difference. This difference is likely the result of division of the nucleus in SLN microspores. The average percentage of MN microspores increases between days 3 and 6 (Figure 5), and the division of nuclei in SLN microspores likely causes this increase. If some nuclei from SLN microspores divide and form MN microspores between days 3 and 6, then the observed rate of increase in SLN microspores will decrease between days 3 and 6 as the average percentage of MN microspores increases, which is exactly what we see. The respective rates of change in BN and SLN microspores, therefore, are likely similar throughout the six-day test period despite the 3.03\% difference between days 3 and 6 .

Although nuclear fusion likely serves as the primary mechanism for chromosome doubling in wheat, we cannot assume it is the only mechanism. Endoreduplication and other mechanisms may account for a small percentage of DH production. Nevertheless, the data provided in this study indicate that nuclear fusion may serve as the primary mechanism for chromosome doubling in wheat microspore embryogenesis.

In summary, data collected in this study indicates nuclei in binucleated microspores fuse to produce doubled haploid microspores containing a single enlarged nucleus. If endoreduplication led to significant amounts of chromosome doubling, then we would expect to see one of the two patterns depending on the cell-type in which endoreduplication took place. If endoreduplication occurred in SSN microspores, then the percentage of SSN microspores would decrease over time while SLN microspores increased. If endoreduplication occurred in BN microspores, then the percentage of BN microspores would remain stable over time, because both nuclei would remain present in the cell while one or both would increase in size. Because the average percentage of SSN microspores remained steady over time, and because the average percentage of BN microspores decreased at a rate 
similar to the rate of increase of SLN microspores, the data from this study indicated that nuclear fusion may serve as one of the mechanisms for chromosome doubling. In conclusion, the proportion of microspores containing enlarged nuclei during the first week of induction culture may be used to predict the relative success of microspore embryogenesis; thus making the earlier evaluation of microspore culture efficiency reliable. A reliable early assessment of microspore cultures will serve to save cost and make the production of doubled haploids more efficient.

\section{References}

[1] Touraev, A., Vicente, O. and Heberle-Bors, E. (1997) Initiation of Microspore Embryogenesis by Stress. Trends in Plant Science, 2, 297-302. http://dx.doi.org/10.1016/S1360-1385(97)89951-7

[2] Gervais, C., Newcomb, W. and Simmonds, D.H. (2000) Rearrangement of the Actin Filament and Microtubule Cytoskeleton during Induction of Microspore Embryogenesis in Brassica napus L. cv. Topas. Protoplasma, 213, 194-202. http://dx.doi.org/10.1007/BF01282157

[3] Liu, W., Zheng, M.Y., Polle, E.A. and Konzak, C.F. (2002) Highly Efficient Doubled-Haploid Production in Wheat (Triticum aestivum L.) via Induced Microspore Embryogenesis. Crop Science, 42, 686-692. http://dx.doi.org/10.2135/cropsci2002.0686

[4] Maraschin, S.D.F., De Priester, W., Spaink, H.P. and Wang, M. (2005) Androgenic Switch: An Example of Plant Embryogenesis from the Male Gametophyte Perspective. Journal of Experimental Botany, 56, 1711-1726. http://dx.doi.org/10.1093/jxb/eri190

[5] Touraev, A., Indrianto, A., Wratschko, I., Vicente, O. and Heberle-Bors, E. (1996) Efficient Microspore Embryogenesis in Wheat (Triticum aestivum L.) Induced by Starvation at High Temperature. Sexual Plant Reproduction, 9, 209215. http://dx.doi.org/10.1007/BF02173100

[6] Zheng, M., Liu, W., Weng, Y., Polle, E. and Konzak, C. (2001) Culture of Freshly Isolated Wheat (Triticum aestivum L.) Microspores Treated with Inducer Chemicals. Plant Cell Reports, 20, 685-690. http://dx.doi.org/10.1007/s00299-001-0393-0

[7] Maraschin, S.D.F., Vennik, M., Lamers, G.E., Spaink, H.P. and Wang, M. (2005) Time-Lapse Tracking of Barley Androgenesis Reveals Position-Determined Cell Death within Pro-Embryos. Planta, 220, 531-540. http://dx.doi.org/10.1007/s00425-004-1371-x

[8] Touraev, A., Pfosser, M., Vicente, O. and Heberle-Bors, E. (1996) Stress as the Major Signal Controlling the Developmental Fate of Tobacco Microspores: Towards a Unified Model of Induction of Microspore/Pollen Embryogenesis. Planta, 200, 144-152. http://dx.doi.org/10.1007/bf00196662

[9] Zaki, M.A.M. and Dickinson, H.G. (1990) Structural Changes during the First Divisions of Embryos Resulting from Anther and Free Microspore Culture in Brassica napus. Protoplasma, 156, 149-162. http://dx.doi.org/10.1007/BF01560653

[10] Zheng, M.Y., Liu, W., Weng, Y., Polle, E. and Konzak, C.F. (2003) Production of Doubled Haploids in Wheat (Triticum aestivumL.) through Microspore Embryogenesis Triggered Byinducer Chemicals. In: Maluszynski, M., Kasha, K., Forster, B.P. and Szarejko, I., Eds., Doubled Haploids Production in Crop Plants, A Manual, Kluwer Academic Publisher, Dordrecht, 83-94.

[11] Hu, T. and Kasha, K.J. (1999) A Cytological Study of Pretreatments Used to Improve Isolated Microspore Cultures of Wheat (Triticum aestivum L.) cv. Chris. Genome, 42, 432-441.

[12] Kasha, K.J., Simion, E., Oro, R., Yao, Q.A., Hu, T.C. and Carlson, A.R. (2002) An Improved in Vitro Technique for Isolated Microspore Culture of Barley. In: Maluszynski, M. and Kasha, K.J., Eds., Mutations, In Vitro and Molecular Techniques for Environmentally Sustainable Crop Improvement, Springer, Netherlands, 45-54. http://dx.doi.org/10.1007/978-94-015-9996-2_5

[13] Zheng, M.Y. (2003) Microspore Culture in Wheat (Triticum aestivum)—Doubled Haploid Production via Induced Embryogenesis. Plant Cell, Tissue and Organ Culture, 73, 213-230. http://dx.doi.org/10.1023/A:1023076213639

[14] Kasha, K.J., Hu, T.C., Oro, R., Simion, E. and Shim, Y.S. (2001) Nuclear Fusion Leads to Chromosome Doubling during Mannitol Pretreatment of Barley (Hordeum vulgare L.) Microspores. Journal of Experimental Botany, 52, 1227-1238. http://dx.doi.org/10.1093/jexbot/52.359.1227

[15] Joubès, J. and Chevalier, C. (2000) Endoreduplication in Higher Plants. In: Inzé, D., Ed., The Plant Cell Cycle, Springer, Netherlands, 191-201. http://dx.doi.org/10.1007/978-94-010-0936-2 15

[16] Sunderland, N., Collins, G.B. and Dunwell, J.M. (1974) The Role of Nuclear Fusion in Pollen Embryogenesis of Datura innoxia Mill. Planta, 117, 227-241. http://dx.doi.org/10.1007/BF00388396

[17] Sunderland, N. (1974) Anther Culture as a Means of Haploid Induction. In: Kasha, Ed., Haploids in Higher Plants: 
Advances and Potential, University of Guelph, Guelph, 91-122.

[18] Chen, C.C., Howarth, M.J., Peterson, R.L. and Kasha, K.J. (1984) Ultrastructure of Androgenic Microspores of Barley during the Early Stages of Anther Culture. Canadian Journal of Genetics and Cytology, 26, 484-491. http://dx.doi.org/10.1139/g84-076

[19] Testillano, P., Georgiev, S., Mogensen, H.L., Coronado, M.J., Dumas, C., Risueño, M.C. and Mathys-Rochon, E. (2004) Spontaneous Chromosome Doubling Results from Nuclear Fusion during in Vitro Maize Induced Microspore Embryogenesis. Chromosoma, 112, 342-349. http://dx.doi.org/10.1007/s00412-004-0279-3

[20] González-Melendi, P., Ramírez, C., Testillano, P.S., Kumlehn, J. and Risueño, M.C. (2005) Three Dimensional Confocal and Electron Microscopy Imaging Define the Dynamics and Mechanisms of Diploidisation at Early Stages of Barley Microspore-Derived Embryogenesis. Planta, 222, 47-57. http://dx.doi.org/10.1007/s00425-005-1515-7

[21] Shim, Y.S., Kasha, K.J., Simion, E. and Letarte, J. (2006) The Relationship between Induction Ofembryogenesis and Chromosome Doubling in Microspore Cultures. Protoplasma, 228, 79-86. http://dx.doi.org/10.1007/s00709-006-0177-Z

[22] Liu, W., Zheng, Y. and Konzak, C. (2002) Improving Green Plant Production via Isolated Microspore Culture in Bread Wheat (Triticum aestivum L.). Plant Cell Reports, 20, 821-824. http://dx.doi.org/10.1007/s00299-001-0408-X

[23] Zheng, M., Weng, Y., Liu, W. and Konzak, C. (2002) The Effect of Ovary-Conditioned Medium on Microspore Embryogenesis in Common Wheat (Triticum aestivum L.). Plant Cell Reports, 20, 802-807. http://dx.doi.org/10.1007/s00299-001-0411-2

[24] Carey, G. (1998) Multivariate Analysis of Variance (MANOVA): I. Theory. Academic Press, Boston.

[25] Martínez-Pérez, E., Shaw, P., Reader, S., Aragón-Alcaide, L., Miller, T. and Moore, G. (1999) Homologous Chromosome Pairing in Wheat. Journal of Cell Science, 112, 1761-1769.

[26] Martínez-Pérez, E., Shaw, P. and Moore, G. (2001) The Ph1 Locus Is Needed to Ensure Specific Somatic and Meiotic Centromere association. Nature, 411, 204-207. http://dx.doi.org/10.1038/35075597

\section{Abbreviations}

Microspore containing a single small nucleus (SSN),

Microspore containing a single large nucleus (SLN),

Binucleated microspore (BN),

Multinucleated microspore containing three or more nuclei (MN),

Binucleated microspore containing two small nuclei (BNS),

Binucleated microspore containing at least one large nucleus (BNL),

Multinucleated microspore containing small nuclei (MNS),

Multinucleated microspore containing at least one large nucleus (MNL),

4',6-diamidino-2-phenylindole (DAPI). 